\title{
As CARACTERÍSTICAS DERMATOGLÍFICAS DE CRIANÇAS E ADOLESCENTES TALENTOSOS DO INSTITUTO Rogério Steinberg do Rio de Janeiro - RJ
}

\author{
Renato Vidal Linhares* \\ José Fernandes Filho** \\ Marsyl Bulkool Mettrau***
}

\section{Resumo}

A identificação de pessoas talentosas é um dos pontos mais difíceis, além de ser mal desenvolvido no Brasil. O objetivo deste artigo foi identificar o perfil dermatoglífico de jovens considerados talentosos pelo Instituto Rogério Steinberg - RJ. O conhecimento do perfil de um grupo facilita o seu desenvolvimento, a descoberta de suas potencialidades e cria índices que norteiam a detecção de futuros talentos. No caso da amostra pesquisada, observou-se que essas crianças e adolescentes possuem um baixo percentual de desenhos tipo arco e altos níveis de deltas e de linhas nos dez dedos das mãos, características estas similares às encontradas em atletas de alto rendimento. Além disso, apresentaram o fenômeno de espelho entre as mãos, ou seja, uma similaridade nos desenhos digitais e na relação do número de linhas entre os dedos das mãos correspondentes quase perfeitas, como a hipótese sugerida neste estudo. Logo, isso nos leva a acreditar que a dermatoglifia pode ser uma ferramenta auxiliativa na identificação de indivíduos talentosos.

Palavras-chave: perfil; dermatoglifia; altas habilidades; jovens.

* Universidade Federal do Rio de Janeiro, Rio de Janeiro, RJ, Brasil; Universidade Salgado de Oliveira, Niterói, RJ, Brasil.

** Universidade Federal do Rio de Janeiro, Rio de Janeiro, RJ, Brasil.

*** Universidade Salgado de Oliveira, Niterói, RJ, Brasil. 


\begin{abstract}
THE DERMATOGLYPHICS CHARACTERISTICS OF THE TALENTED CHILDREN AND ADOLESCENTS OF INSTITUTE ROGÉRIO STEINBERG OF RIO DE JANEIRO - RJ

The identification of talented people is one of the most difficult and has been badly done in Brazil. The aim of this paper was to identify the dermatoglyphic profile of young talented considered by the Instituto Rogério Steinberg - RJ. In the case of the sample surveyed, was observed that these children and adolescents have a low percentage of arch type designs and high levels of deltas and lines in the ten fingers of the hands, characteristic similar to those found in high-performance athletes. Moreover, they presented a mirror relation between the hands, is a similarity in digital designs and the relationship of the number of lines between the fingers almost perfect matching, as found in high-performance athletes. Therefore, the dermatoglyphics may be more useful evaluative tool in the identification of talented individuals.
\end{abstract}

Keywords: profile; dermatoglyphic; gifted; young.

\title{
Resumen
}

LAS CARACTERÍSTICAS DERMATOGLÍFICAS DE NIÑOS Y ADOLESCENTES TALENTOSOS DEL INSTITUTO ROGÉRIO STEINBERG DE RIO DE JANEIRO - RJ

La identificación de personas talentosas es uno de los puntos más difíciles y ha sido mal trabajado en Brasil. El objetivo de este artículo fue identificar el perfil dermatoglífico de jóvenes considerados talentosos por el Instituto Rogério Steinberg - RJ. El conocimiento del perfil de un grupo facilita su desarrollo y el descubrir sus potencialidades crea indicadores que orientaran la detección de futuros talentos. En el caso de la muestra investigada se observó que esos niños y adolescentes poseen un bajo porcentaje de diseños tipo arco y altos niveles de deltas y de líneas en los diez dedos de las manos; características similares a las encontradas en deportistas de alto rendimiento. Además, se observa el fenómeno de espejo entre las manos, es decir, una similitud en los diseños digitales y en la relación del número de líneas entre los dedos de las manos correspondientes casi perfectas, como la hipótesis sugerida en este estudio. Por lo tanto, esto nos lleva a pensar que la dermatoglifía puede ser una herramienta complementaria en la identificación de individuos talentosos.

Palavras clave: perfil; dermatoglifía; altas habilidades; jóvenes. 


\section{Introduçáo}

Indivíduos descritos com altas habilidades são aqueles que em qualquer campo do saber ou do fazer que possam ser observados e/ou relatados possuem "traços consistentemente superiores" em relação a uma média (Brasil, MEC/ SEESP, 1995). O Conselho Europeu também utiliza o termo Hight Hability (Altas Habilidades) e o Conselho Mundial o termo Gifted (Superdotação) ou talentos (Reis, 2006). Pessoas que apresentam um modus operandi extremamente diferenciado são geralmente reconhecidas com características de altas habilidades (Renzulli, 2004), sendo adotado o termo "altas habilidades" (AH) para identificá-los enquanto um grupo especial.

Um dos pontos que merece destaque no trabalho com esse grupo especial é a sua identificação, pois há uma dificuldade na normatização de critérios de identificação (Solow, 2001), ou seja, há a necessidade de métodos avaliativos que permitam a percepção consistente de "traços superiores" no indivíduo. As ações acima da média que permanecem com frequência e duração no repertório dos comportamentos de uma pessoa e que podem também ser registradas em momentos diferentes e situações semelhantes são chamadas de "traços" (Brasil, MEC/SEESP, 1995). De acordo com a Secretaria de Educação Especial (Brasil, MEC/SEESP, 1995), os alunos devem ser observados na sua totalidade, sendo que a utilização de vários tipos de avaliaçóes facilita a identificação dos talentos (Fleith, 1999).

Renzulli (2004) descreve que habilidade acima da média, envolvimento com a tarefa e criatividade são os três comportamentos que identificam pessoas altamente produtivas, sendo que para Mettrau e Reis (2007) e Brasil (Brasil, MEC/ SEESP, 1995) há uma grande importância na identificação de pessoas talentosas o quanto mais cedo possível, assim como dos aspectos que as condicionam (Garcia, 2005), pois assim um melhor trabalho de enriquecimento pode ser realizado pelos professores.

Atualmente há um crescimento no número de entidades (ABAHSD, IRS, CONBRASD, ASPAT, APAHSD, PESTALOZZI entre outras) voltadas ao apoio de jovens com altas habilidades e/ou de seus familiares. A atenção a esse grupo especial é de grande importância para a sociedade, pois o seu desenvolvimento pode gerar soluçóes para as necessidades da população e consequentemente contribuir socialmente (Mettrau \& Reis, 2007).

Para Fingelkurts e Fingelkurts (2003) uma pessoa com Altas Habilidades não nasce pronta, mas o seu potencial é inato, ou seja, os talentosos possuem potencialidades genéticas, as quais sob influência dos fatores externos (ambiente) 
determinarão o ritmo e o grau de desenvolvimento do indivíduo (Filin \& Volkov, 1998). De acordo com Relvas (2008), dentre as várias definiçóes existentes sobre a inteligência, a capacidade de aprender é a mais utilizada pelos autores, sendo considerada de origem largamente genética, relativamente fixa e constante (Fonseca, 2007). Além disto, Plomin e Spinath (2004) consideram que no futuro pós-genoma as pesquisas genéticas ligadas à inteligência e outras áreas da personalidade aumentarão.

Ao relacionarmos o comportamento acima da média descrito por Renzulli (2004) e a potencialidade genética inata relatada anteriormente, acredita-se que é possível através de métodos avaliativos genéticos encontrarmos formas de identificar esse grupo especial de pessoas. Freeman e Guenther (2000), citando os estudos de Plomin et al. (1994) e Bouchard (1997) com gêmeos idênticos e não-idênticos separados ao nascer, relatam que foi encontrada uma influência da genética na inteligência na ordem de 70\%. Knackfuss, Accioly Júnior e Fernandes Filho (2005), ao estudarem o perfil dermatoglífico entre três geraçóes, encontraram características genéticas similares entre elas.

No esporte atualmente é utilizada uma ferramenta avaliativa que possibilita a observação de potencialidades genéticas, a qual é descrita como método dermatoglífico, o qual estuda os padróes das digitais dos dedos das mãos, da palma da mão e da sola do pé (Matsuyama \& Ito, 2006). A dermatoglifia (do latim, dermo, significando "pele"; e do grego, glypha, "gravar") é um termo proposto por Cummins e Midlo que foi introduzido em 1926 na 42a Sessão Anual da Associação Americana de Anátomos. A dermatoglifia analisa as características das impressóes digitais, sendo considerado um marcador genético que pode ser observado desde o nascimento, sendo praticamente imutável (Mercanti, Bezerra, Fernandes Filho \& Struchiner, 2004), além de ser uma avaliação de fácil realização e baixo custo.

Atualmente há um grande número de estudos sobre perfis dermatoglíficos de atletas de alto rendimento (Domingues, Dantas, \& Fernandes Filho, 2003; Dantas, Alonso \& Fernandes Filho, 2005; Fernandes \& Fernandes Filho, 2004; Dantas, 2004; Tuche, Fazolo, Assis, Dantas, \& Fernandes Filho, 2005; Carvalho, Fernandes Filho, \& Novaes, 2005; Ferreira \& Fernandes Filho, 2006; Fonseca, Dantas, Fernandes, \& Fernandes Filho, 2008), sendo que um dos principais pontos interessantes no estudo das digitais como ferramenta avaliativa no auxílio da identificação de indivíduos com potencial para altas habilidades refere-se ao fenômeno de espelho entre as mãos. De acordo com Abramova, Nikitina e Ozolin (1996) as impressóes digitais sofrem modificações nos seus índices de acordo com os diferentes níveis de qualificação esportiva, 
sendo que atletas de alto rendimento demonstraram nos estudos de Ferreira e Fernandes Filho (2006) e Dantas (2004) uma relação de espelho no tipo de desenho e no número de linhas entre os dedos das mãos, nos levando a acreditar que a mesma relação de espelho entre as mãos encontradas em atletas de alta qualificação esportiva, ou seja, um grupo especial de pessoas com altas habilidades esportivas, possa também ser encontrado em outras áreas do saber e fazer em indivíduos com capacidades acima da média.

Com isso, este estudo buscou avaliar pessoas com altas habilidades em áreas que não sejam esportivas em relação às suas características dermatoglíficas. Para isso, utilizou-se como amostra um grupo de crianças e adolescentes identificados como talentosos do Instituto Rogério Steinberg, Rio de Janeiro - RJ.

\section{Metodologia}

\section{Amostra}

A amostra foi composta de 25 crianças e adolescentes diagnosticados com altas habilidades em diferentes áreas do conhecimento pelo Instituto Rogério Steinberg - RJ, sendo que o instituto foi escolhido por ser reconhecido socialmente. Todos os alunos fazem parte da Oficina de Criação da instituição e entregaram o termo de consentimento informado assinado pelos responsáveis.

Os alunos que participam da Oficina de Criação já foram diagnosticados como sendo talentosos pela instituição, por isso foram incluídos na amostra. As crianças e adolescentes pesquisados possuem entre 8 e 14 anos com média e desvio padrão de $11,8 \pm 1,71$ anos.

\section{Procedimentos}

Inicialmente houve uma descrição das características da pesquisa e dos motivos que nos levaram ao interesse na sua realização. Em seguida receberam o Termo de Autorização do Responsável para que pudessem participar da pesquisa. Posteriormente foi marcada uma data no próprio Instituto para a realização da avaliação dermatoglífica, da qual só participaram os alunos que trouxeram a autorização assinada pelo responsável. Para finalizar, houve a análise das impressóes digitais e a tabulação dos dados. 


\section{Instrumentos}

Para a coleta dos dermatóglifos dos dedos das mãos utilizou-se uma almofada coletora de impressóes digitais (Impress ${ }^{\circledR}$ ) e papel branco rugoso, sendo o método para a análise o relatado por Cummins e Midlo (1961), com a observação dos três tipos de desenhos existentes: tipo arco, composto de cristas que atravessam transversalmente a digital, não possui deltas e é representado pela letra "A"; tipo presilha, que corresponde a um feixe de linhas paralelas que faz uma volta de 180 graus, possui sempre um delta e é representado pela letra "L"; e verticilo, sistema nuclear de linhas formando círculos concêntricos ou espirais possuindo dois deltas, um de cada lado, e é representado pela letra "W". A contagem de linhas (cristas papilares) foi realizada traçando-se uma linha que une o delta ao centro do sistema nuclear, sendo que nessa contagem não se incluem as linhas que constituem o centro do sistema nuclear e do delta. No desenho tipo arco não se faz a contagem de linhas, pois o mesmo náo possui delta. Nos desenhos verticilos têm-se duas contagens, uma para cada delta, sendo considerado como número de linhas a média das duas contagens, adicionando-se uma linha, caso o total seja número ímpar. A soma total do número de linhas nos dez dedos das mãos corresponde ao SQTL. Outro índice dermatoglífico analisado refere-se ao número total de deltas dos dez dedos das mãos (D10). Na figura abaixo é possível observar as principais diferenças entre os três tipos de desenhos que são: no desenho tipo arco não há deltas, na presilha há a presença de um delta e no verticilo há dois deltas.

Figura 1. Tipos de desenhos dermatoglíficos

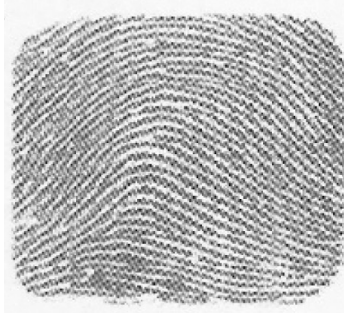

Figurat - Arco $(A)$

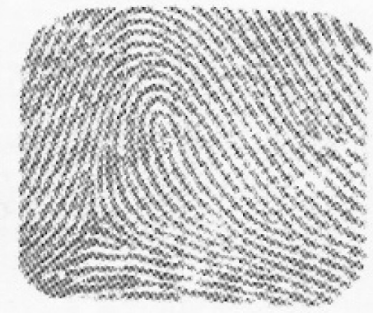

Figura 11 - Presitha (L)

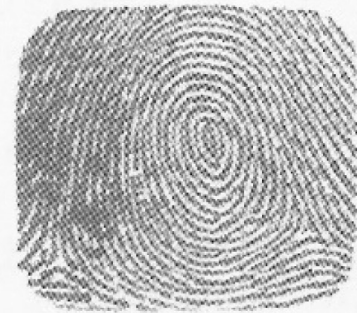

Figura III - Vertículo (W) 


\section{Resultados}

Os resultados relacionados ao perfil que identifica as principais características dos alunos do IRS estão demonstrados nas tabelas 1, 2 e 3 apresentadas a seguir.

$\mathrm{Na}$ tabela 1, estão os percentuais dos tipos de desenhos dermatoglíficos, a quantidades de deltas (D10) e o somatório da quantidade de linhas nos dez dedos das mãos (SQTL). Os alunos apresentaram um percentual maior de desenhos do tipo presilha (68\%), seguido de verticilo (24\%) e uma porção menor de arcos $(08 \%)$.

\begin{tabular}{|l|c|c|c|c|c|}
\hline Caracteres & $\mathrm{A}(\%)$ & $\mathrm{L}(\%)$ & $\mathrm{W}(\%)$ & $\mathrm{D} 10$ & SQTL \\
\hline Média & 08 & 68 & 24 & 11,6 & 116,3 \\
\hline Mínimo & 00 & 02 & 00 & 03 & 09 \\
\hline Máximo & 07 & 10 & 08 & 18 & 199 \\
\hline
\end{tabular}

As tabelas seguintes referem-se aos resultados médios, mínimos e máximos dos valores dos tipos de desenhos de cada dedo da máo esquerda (MET) e da mão direita (MDT) (tabela 2) e da quantidade de linhas de cada dedo da mão esquerda (MESQL) e da mão direita (MDSQL) (tabela 3).

Os resultados nas tabelas 2 e 3 são interessantes e estão de acordo com o esperado pela pesquisa, pois, como no esporte de alto rendimento, foi encontrada uma relação de espelho entre as mãos, tanto no tipo de desenho como na configuração do número de linhas de cada dedo das mãos, como é possível verificar a partir das setas. A única exceção foi quanto ao tipo de desenho, pois houve uma igualdade nos valores médios do primeiro para o segundo dedo, enquanto na mão esquerda houve uma queda entre esses dedos.

Tabela 2. Resultados médios, mínimo e máximo dos tipos de desenhos de cada dedo das mãos esquerda e direita.

\begin{tabular}{|l|c|c|c|c|c|c|c|c|c|c|}
\hline Caracteres & MET & MET & MET & MET & MET & MDT & MDT & MDT & MDT & MDT \\
Média & 1,2 & $\downarrow 1,1$ & $\downarrow 1$ & $\uparrow 1,2$ & $\downarrow 0,9$ & 1,2 & $=1,2$ & $\downarrow 1,1$ & $\uparrow 1,3$ & $\downarrow 1$ \\
\hline Mínimo & 00 & 00 & 00 & 00 & 00 & 00 & 00 & 00 & 00 & 00 \\
\hline Máximo & 02 & 02 & 02 & 02 & 02 & 02 & 02 & 02 & 02 & 02 \\
\hline
\end{tabular}


Tabela 3. Resultados médios, mínimo e máximo do número de linhas de cada dedo das mãos esquerda e direita.

\begin{tabular}{|l|c|c|c|c|c|c|c|c|c|c|}
\hline Caracteres & MES- & MES- & MES- & MES- & MES- & MDS- & MDS- & MDS- & MDS- & MDS- \\
& 1 & 2 & 3 & 4 & 5 & 1 & 2 & 3 & 4 & 5 \\
\hline Média & 12,5 & $\downarrow 10,6$ & $\uparrow 11,0$ & $\uparrow 13,7$ & $\downarrow 10,4$ & 13,8 & $\downarrow 9,6$ & $\uparrow 10,4$ & $\uparrow 12,4$ & $\downarrow 11,6$ \\
\hline Mínimo & 00 & 00 & 00 & 00 & 00 & 00 & 00 & 00 & 00 & 00 \\
\hline Máximo & 23 & 16 & 20 & 28 & 23 & 24 & 20 & 19 & 21 & 22 \\
\hline
\end{tabular}

\section{Discussão}

Para Reis (2006) a utilização de testes para a identificação de alunos com altas habilidades é complexa, sendo a caracterização, também chamada de sondagem, o primeiro processo que indica as altas habilidades (Brasil, 1999). O conhecimento do perfil de um grupo facilita na elaboração dos melhores meios para o seu desenvolvimento (Bolsoni-Silva, Paiva, \& Barbosa, 2009) e na descoberta das suas potencialidades (Mettrau, 1999), criando índices que norteiam na detecção de futuros talentos (Menezes \& Fernandes Filho, 2006).

Observando os resultados da tabela 1 quanto ao percentual dos tipos de desenhos digitais, os quais são analisados em associação às qualidades físicas básicas (Dantas et al., 2004), foi possível destacar que os alunos talentosos do IRS possuem menores índices de desenhos tipo Arco (8\%), característica esta similar aos atletas de alto rendimento (Santos \& Fernandes Filho, 2004; Dantas, 2004), já que essa digital relaciona-se a baixa potencialidade coordenativa (Linhares, Matta, Lima, Dantas, Costa, \& Fernandes Filho, 2009). Em relação ao desenho tipo Presilha, o qual se apresentou em maior evidência (68\%), o mesmo demonstra uma relação com atividades que exigem a predisposição para a qualidade física de velocidade (Fazolo et al., 2005; Silva, Vianna, Gomes, \& Dantas, 2008). De forma secundária, sobressaiu o desenho do tipo Verticilo (24\%), que é uma característica de atletas com potencialidade voltada para a qualidade física de resistência ou potência aeróbica, como demonstrado nos resultados encontrados por Almeida, Fernandes Filho e Dantas (2005), ou seja, possui relação com atividades cíclicas. 
Outra similaridade encontrada entre os índices dermatoglíficos de atletas de alto rendimento e dos alunos do IRS é no número de D10 e SQTL, pois a associação do baixo nível de arcos com altos níveis de D10 e SQTL estão relacionados à alta potencialidade coordenativa (Dantas et al., 2005), sendo também um índice característico de atletas profissionais. Como exemplo, os resultados encontrados nas crianças e adolescentes do IRS são similares a atletas profissionais de futebol de campo (Fernandes \& Fernandes Filho, 2004), atletas da seleção brasileira de basquete masculina campeã do Pan-Americano de 2003 (Domingues et al., 2003), atletas da seleçáo brasileira feminina de vôlei de 2004 (Fonseca et al., 2008), atletas de ciclismo de estrada masculino elite do Brasil (Tuche et al., 2005), atletas de alto rendimento participantes de corrida de resistência do Rio de Janeiro (Carvalho et al., 2005) entre outras modalidades esportivas de alta qualificação.

Nas tabelas 2 e 3 foram descritos os resultados médios relacionados ao tipo de desenho digital e o número de linhas em cada dedo das mãos como forma de se analisar a relação de espelho entre as mãos, ou seja, a similaridade entre os tipos de desenhos dos dedos correspondentes da mão direita e esquerda e no sequenciamento do número de linhas entre um dedo e o seguinte, como encontrado em atletas de alta qualificação (Ferreira \& Fernandes Filho, 2006; Dantas, 2004; Fernandes \& Fernandes Filho, 2004).

De acordo com os resultados encontrados nos alunos talentosos do IRS avaliados é possível constatar o mesmo fenômeno de espelho entre as mãos. $\mathrm{Na}$ tabela 2, na qual está demonstrada a simetria quanto ao tipo de desenho digital, é possível observar que essa relação é quase perfeita, com exceção do primeiro para o segundo dedos, onde se encontrou valores médios iguais na mão direita e uma queda na mão esquerda. Nos resultados referentes ao número de linhas a simetria foi perfeita, ou seja, do primeiro para o segundo dedos houve queda no número de linhas, do segundo para o terceiro e do terceiro para o quarto houve aumento e do quarto para o quinto aconteceu uma queda.

\section{Conclusáo}

Conclui-se através dos resultados deste estudo que os mesmos estão de acordo com o esperado, demonstrando que os alunos talentosos do IRS possuem um padrão de similaridade entre os desenhos digitais e o número de linhas entre os dedos das mãos (espelho entre as mãos) quase perfeitas como encontrado nos esportistas de alta qualificação. Isso nos leva a crer que o método dermatoglífico 
pode ser mais uma ferramenta avaliativa no auxílio da identificação de indivíduos com altas habilidades.

Recomenda-se que outros estudos com pessoas talentosas das mais diversas áreas, assim como com escolares em geral, sejam conduzidos para que os índices encontrados neste estudo sejam comparados e os dados aqui expostos ampliados.

\section{Referências}

Abramova, T. F., Nikitina, T. M., \& Ozolin, N. N. (1996). Impressōes dermatoglíficas Marcas genéticas no potencial energético do homem. Anais Científicos do ano de 1995, Moscou.

Almeida, M. N., Fernandes Filho, J., \& Dantas, P. M. S. (2005). Relação dos índices dermatoglíficos com avaliação isocinética e ergoespirometria. Fitness \& Performance Journal, 4(2), 240-249.

Bolsoni-Silva, A. T., Paiva, M. M., \& Barbosa, C. G. (2009). Problemas de comportamento de crianças/adolescentes e dificuldades de pais/cuidadores: um estudo de caracterização. Psicologia Clínica, 21(1), 169-184.

Brasil. Ministério da Educação. Secretaria de Educação Especial. (1999). Programa de capacitação de recursos humanos do ensino fundamental: superdotaçáo e talento, v. 1. Brasília.

Brasil. Ministério da Educação. Secretaria da Educação Especial. (1995). Subsidios para organização e funcionamento de serviços de educação especial: área de deficiência. Brasília. (Séries Diretrizes, 9).

Carvalho, E., Fernandes Filho, J., \& Novaes, J. S. (2005). Perfis dermatoglífico, somatotípico e fisiológico dos atletas de alto rendimento, participantes de corrida de resistência no Rio de Janeiro. Fitness e Performance Journal, 4(3), 168-174.

Cummins, H., \& Midlo, C. (1961). Finger prints, palms and soles an introduction to dermatoglyphics. Philadelphia: Blakiston.

Dantas, P. M. S. (2004). Relação entre estado e predisposição genética no futsal brasileiro. (Tese de Doutorado. Programa de Pós-graduaçáo da Universidade Federal do Rio Grande do Norte, Natal, RN).

Dantas, P. M. S., Alonso, L., \& Fernandes Filho, J. (2005). Características dermatoglíficas, de qualidades físicas básicas e somatotípicas no futsal em três níveis de qualificação (alto rendimento, rendimento intermediário e baixo rendimento). In E. H. M. Dantas \& J. Fernandes Filho (Eds.), Atividade fisica em ciências da saúde (pp. 109-124). Rio de Janeiro: Shape. 
Dantas, P. M. S., Fernandes, P. R., Ganime, F. B., Ribeiro, E. M., Brüch, V. L., Caruso, P., Batista, L. A., \& Fernandes Filho, J. (2004). Dermatoglyphics characteristics of the brazilian high performance athletes of sportive modalities. Journal of the International Federation of Physical Education, 74(1), 124-128.

Domingues, E. A. F., Dantas, P. M. S., \& Fernandes Filho, J. (2005). Identificação dos perfis dermatoglífico e somatotípico dos atletas da seleção brasileira de basquete adulto masculino, campeã Pan-Americana, 2003. Fitness \& Performance Journal, 4(1), 72-80.

Fazolo, E., Cardoso, P. G., Tuche, W., Menezes, I. C., Teixeira, M. E. S., Portal, M. N. D., Nunes, R. M. A., Costa, G., Dantas, P. M. S., \& Fernandes Filho, J. (2005). A dermatoglifia e a somatotipologia no alto rendimento do Beach Soccer - Seleçáo Brasileira. Revista de Educação Física, 130, 45-51.

Fernandes, P. R., \& Fernandes Filho, J. (2004). Estudo comparativo da dermatoglifia, somatotipia e do consumo máximo de oxigênio dos atletas da seleçáo brasileira de futebol de campo, portadores de paralisia cerebral e de atletas profissionais de futebol de campo, náo portadores de paralisia cerebral. Fitness \& Performance Journal, 3(3), 157-165.

Ferreira, H. B., \& Fernandes Filho, J. (2006). O perfil dos níveis de força e dermatóglifos dos atletas da seleção brasileira de canoagem slalom. Lecturas, Educación Física y Deportes, Revista Digital, 13(123). Recuperado em 21 de outubro, 2008, de www. efdeportes.com.

Filin, V. P., \& Volkov, V. M. (1998). Seleção de talentos nos esportes. Londrina: Midiograf.

Fingelkurts, A. A., \& Fingelkurts, A. A. (2003). Gifted brain and twinning: integrative review of the recent literature. Advances in Psychology Research, 20, 1-32.

Fleith, D. S. (1999). Psicologia e educação do superdotado: definição, sistema de identificação e modelo de estimulação. Cadernos de Psicologia da SBP, 5(1), 37-50.

Fonseca, V. (2007). Cognição, neuropsicologia e aprendizagem: abordagem neuropsicológica e psicopedagógica. Petrópolis: Vozes.

Fonseca, C. L. T., Dantas, P. M. S., Fernandes, P. R., \& Fernandes Filho, J. (2008). Perfil dermatoglífico, somatotípico e da força explosiva de atletas da seleção brasileira de voleibol feminina. Fitness \& Performance Journal, 7(1), 35-40.

Freeman, J., \& Guenther, Z. C. (2000). Educando os mais capazes: ideias e açôes comprovadas. São Paulo: EPU.

Garcia, R. L. (2005). Predictores de talento. Intangible Capital, 1(7), 1-25.

Knackfuss, M. I., Accioly Jr., H., \& Fernandes Filho, J. (2005). Características dermatoglíficas da cultura do brincar e das qualidades físicas básicas. In E. H. M. Dantas \& J. Fernandes Filho (Eds.), Atividade física em ciências da saúde (pp. 15-28). Rio de Janeiro: Shape.

Linhares, R. V., Matta, M. O., Lima, J. R. P., Dantas, P. M. S., Costa, M. B., \& Fernandes Filho, J. (2009). Effects of sexual maturation on body composition, dermatoglyphics, so- 
matotype and basic physical qualities of adolescents. Arquivos Brasileiros de Endocrinologia \& Metabologia, 53(1), 48-54.

Matsuyama, N., \& Ito, Y. (2006). The Frequency of Fingerprint Type in Parents of Children with Trisomy 21 in Japan. Journal of Physiologial Anthropology, 25(1), 15-21.

Menezes, L. S., \& Fernandes Filho, J. (2006). Identificação e comparação das características dermatoglíficas, somatotípicas e qualidades físicas básicas de atletas de GRD de diferentes níveis de qualificação. Fitness \& Performance Journal, 5(6), 393-401.

Mercanti, L. B., Bezerra, M. L. S., Fernandes Filho, J., \& Struchiner, C. J. (2004). Dermatoglifia e composição corporal em apnéia obstrutiva do sono. Arquivos Neuropsiquiátricos, 62(3), 858-864.

Mettrau, M. B (1999). Reflexôes e ações sobre as Altas Habilidades. Revista Mediação do Instituto Helena Antipoff, 1, 15-21.

Mettrau, M. B., \& Reis, H. M. M. S. (2007). Políticas públicas: altas habilidades/superdotação e a literatura especializada no contexto da educação especial/inclusiva. Revista Ensaio: avaliação e políticas públicas em educação, 15(57), 489-509.

Plomin, R., \& Spinath, F. M. (2004). Intelligence: genetics, gene, and genomics. Journal of Personality and Social Psychology, 86(1), 112-129.

Reis, H. M. M. S. (2006). Educação inclusiva é para todos?: a (falta de) formação docente para Altas Habilidades/Superdotação no Brasil. (Tese de Doutorado. Programa de Pós-Graduação da Faculdade de Educação da Universidade Estadual do Rio de Janeiro, Rio de Janeiro, RJ).

Relvas, M. P. (2008). Neurociências e transtornos de aprendizagem: as múltiplas eficiências para uma educação inclusiva. Rio de Janeiro: Wak.

Renzulli, J. S. (2004). A Pratical System for Identifying Gifted and Talented Students. Recuperado em 28 de agosto, 2010, de http://www.gifted.uconn.edu/sem/semart.html.

Solow, R. (2001). Parent's conception of giftedness. Gifted Child Today, 24, 14-22.

Santos, M. R., \& Fernandes Filho, J. (2004). Estudo dos perfis dermatoglífico, somatotípico e das qualidades físicas básicas dos pára-quedistas do exército brasileiro do ano de 2003. Fitness \& Performance Journal, 3(2), 88-99.

Silva, I. A. S., Vianna, M. V. A., Gomes, A. L. M., \& Dantas, E. H. M. (2008). Diagnóstico do potencial genético físico e somatotipia de uma equipe de futebol profissional Fluminense. Revista Brasileira de Futebol, 1(1), 49-58.

Tuche, W., Fazolo, E., Assis, M., Dantas, P. M. S., \& Fernandes Filho, J. (2005). Perfil dermatoglífico e somatotípico de ciclistas de alto rendimento do Brasil. Revista de Educação Fisica, 132, 14-19. 